\title{
Thought Control Strategies and Rumination in Youth with Acute Stress Disorder and Posttraumatic Stress Disorder Following Single-Event Trauma
}

\author{
Richard Meiser-Stedman, PhD, ${ }^{1}$ Alicia Shepperd, BA, Ed Glucksman, FCEM, ${ }^{2}$ Tim Dalgleish, PhD ${ }^{1}$ \\ William Yule, $\mathrm{PhD}^{3}$ and Patrick Smith, $\mathrm{PhD}^{3}$
}

\begin{abstract}
Objective: Certain thought control strategies for managing the intrusive symptoms of posttraumatic stress disorder (PTSD) are thought to play a key role in its onset and maintenance. Whereas measures exist for the empirical assessment of such thought control strategies in adults, relatively few studies have explored how children and adolescents manage posttraumatic intrusive phenomena.

Methods: In a prospective longitudinal study of 10-16-year-olds with PTSD, who were survivors of road traffic collisions and assaults, a variety of thought control strategies were assessed in the acute phase. These included strategies thought to be protective (reappraisal, social support) as well as maladaptive (distraction, punishment, worry). Ruminative responses to the trauma were assessed at the follow-up assessment.

Results: Posttraumatic stress symptoms (PTSS) at each assessment were associated with the use of punishment and reappraisal, whereas social support and rumination were associated with PTSS symptoms at follow-up. Distraction was unrelated to PTSS at any time point. Rumination accounted for variance in PTSS symptoms at follow-up, even when accounting for baseline PTSS, and was found to mediate the relationships between reappraisal and punishment at baseline and PTSS at the follow-up assessment.

Conclusions: The present study found no evidence to support advocating any particular thought control strategy for managing the intrusive symptoms of PTSD in youth in the acute posttrauma phase, and raised concerns over the use of reappraisal coping strategies. The study underscores the importance of ruminative responses in the onset and maintenance of PTSD in traumaexposed youth.
\end{abstract}

\section{Introduction}

$\mathbf{T}$

He MAIN SymPtoms OF POSTTRAumatiC STRESS DISORDER (PTSD) are distressing, intrusive thoughts, feelings, and images through which the individual relives the traumatic event. Cognitive models of PTSD (e.g., Brewin et al. 1996; Ehlers and Clark 2000) propose that some behaviors and cognitive strategies for managing these intrusive phenomena have an important role in the onset and maintenance of posttraumatic stress symptoms (PTSS). The use of maladaptive strategies for managing intrusive trauma-related memories, such as those involving cognitive avoidance (e.g., thought suppression or distraction) or perseverative thinking (e.g., worry or rumination in response to the intrusion) are hypothesised to impede the processing of traumatic memories and to maintain symptoms.
The empirical study of such maladaptive coping has been greatly advanced by the development of a well-standardized measure of thought control strategies, the Thought Control Questionnaire (TCQ; Wells and Davies 1994). The TCQ indexes five strategies for managing intrusions: Distraction, social control (i.e., using peer support and confiding in others about the intrusions), punishment (i.e., self-blame and criticism for the intrusions), reappraisal (i.e., attempts to revaluate and review aspects of the intrusion and the event), and worry (i.e., finding other negative concerns to think about instead). Studies of trauma-exposed adults have found that the use of worry and punishment are correlated with worse PTSS in road traffic collision (RTC) survivors assessed in the acute phase (Warda and Bryant 1998), female survivors of childhood sexual abuse (Scarpa et al. 2009) and treatment-seeking combat-exposed military personnel (Pietrzak et al. 2011), whereas adults with a

\footnotetext{
${ }^{1}$ MRC Cognition \& Brain Sciences Unit, Cambridge, United Kingdom.

${ }^{2}$ King's College Hospital, London, United Kingdom.

${ }^{3}$ Department of Psychology, Institute of Psychiatry, King's College London, London, United Kingdom.

Funding: This research was funded by a United Kingdom Medical Research Council (MRC) research studentship (G78/6730) awarded to Richard Meiser-Stedman.
} 
primary diagnosis of PTSD were found to score higher on the distraction subscale than a group of depressed adults (Reynolds and Wells 1999).

Less is known, however, about whether such strategies are employed by children and adolescents exposed to trauma, and their impact on the development of PTSS. Three studies have investigated thought control strategies in children and adolescents. In a small sample of youth hospitalized following serious injury, Aaron et al. (1999) found that the use of thought suppression was associated with more severe PTSS. In RTC-exposed youth, Stallard (2003) found that PTSD was associated with distraction, rumination (i.e., a perseverative focus on anxiety or mood disorder symptoms and their meaning, which ultimately worsens negative emotion) and social withdrawal, whereas Ehlers and colleagues (2003) found that the use of rumination and thought suppression 2 weeks posttrauma predicted PTSS at 3 month follow up assessment. These studies attest to the counterproductive use of cognitive avoidance and (in the case of the Stallard and Ehlers studies) ruminative responses. However, they are limited by the reliance on single-item measures or inappropriate measures (e.g., the White Bear Suppression Index, as used by Aaron and colleagues, indexes intrusions as well as suppression).

The present investigation looked at the role of the maladaptive thought control strategies in children and adolescents exposed to both RTC and assault trauma. By adapting the existing adult TCQ measure, the study sought to overcome the methodological weaknesses of earlier studies in this area, as well as to consider whether some thought control strategies (e.g., reappraisal and social support) might be protective against the development of PTSS. Such data will not only aid the refinement of the cognitive theory of PTSD in youth, but also inform the practical guidance given to families in the immediate aftermath of trauma. The study utilized a prospective longitudinal format, with PTSS assessed in the acute phase (i.e., 2-4 weeks posttrauma; hereafter T1) and in the chronic phase (i.e., at 6 months posttrauma; hereafter T2). Based on previous research, we hypothesized that the use of punishment, worry, and distraction would correlate positively with PTSS at T1 and T2, and that reappraisal and social support would negatively correlate with PTSS (i.e., they would be protective against the development of PTSD). As a secondary hypothesis, we sought to duplicate the previous results showing a strong association between rumination and PTSD in youth. Furthermore, we also explored the possibility that some thought control strategies (e.g., punishment, worry), although intended by the young person to help with coping in the acute phase posttrauma, may have encouraged the development of a ruminative response to the trauma that promoted the maintenance of PTSS.

\section{Methods}

\section{Participants}

Children and adolescents 10-16 years of age attending an emergency department (ED) in London following an assault or RTC were invited to participate in the study. Of 343 consecutive attendees at the department, $106(30.9 \%)$ consented to participate in the study at $\mathrm{T} 1$; of these, $99(28.9 \%)$ completed at least one questionnaire battery ( 90 at T1, 67 at T2). Ninety-one completed a structured interview for acute stress disorder (ASD) at T1, and 65 completed a structured interview for PTSD at T2. Fifty-six $(56.6 \%)$ participants had been involved in an assault, whereas $43(43.4 \%)$ participants had been involved in a MVC. Thirty-seven (37.4\%) participants were female. Participants' mean age was $14.0(\mathrm{SD}=1.9)$.

\section{Procedure}

Ethical permission to conduct the study was granted by the Research Ethics Committee of the lead author's home institution (Institute of Psychiatry REC No. 058/01).The informed consent of the young person and main caregiver were required for participation in the study. The assessment included the completion of questionnaires relating to their trauma and a structured clinical interview.

\section{Measures}

PTSD Schedule of the Anxiety Disorders Interview Schedule for the DSM-IV: Child and Parent Version (ADISC). ASD and PTSD were assessed using the relevant schedule of the ADIS-C (Silverman and Albano 1996). The ADIS-C is a structured interview schedule designed for the assessment of anxiety disorders in children and adolescents, and diagnoses are derived from both child and parent reports. Additional items were added to the PTSD schedule of the ADIS-C to assess for the presence of ASD at T1 (see Meiser-Stedman et al. 2007).

TCQ-CA. A child and adolescent version of the TCQ (Wells and Davies 1994) was devised. All 30 items of the adult TCQ were included in the child version, but edited extensively to be understood by a younger population. The five subscales of the TCQ-CA comprise distraction (e.g., "I keep myself busy"), punishment ("I get angry with myself for having the thought or memory"), reappraisal ("I try to come up with a better thought or memory"), social support, and worry ("I think about problems that aren't so important"). A copy of the TCQ-CA is available from the Dr. Meiser-Stedman. This novel measure was initially piloted in a large sample of high school pupils $(n=231$, mean age $=14.5)$; Cronbach's $\alpha$ coefficients for the TCQ-CA subscales were acceptable (distraction $=0.68$, punishment $=0.70$, reappraisal $=0.70$, social support $=0.78$, worry $=0.68$ ). Cronbach's $\alpha$ coefficients in the present sample were also acceptable, with the exception of social support (distraction $=0.65$, punishment $=0.64$, reappraisal $=0.68$, social support $=0.45$, worry $=0.68)$. As three of the social support items were positively worded (e.g., "I ask my friends if they have thoughts or memories like mine") and three were negatively worded (such that they overlapped strongly with distraction or other forms of cognitive avoidance, e.g., "I try not to talk about the thought or memory"), it was decided to use only the three positively worded items. The Cronbach's $\alpha$ for this abbreviated scale was improved (0.59) but still of borderline acceptability; its total score was doubled when making comparisons with other TCQ-CA subscales. Participants could respond "never," "sometimes," "often," or "almost always" to each item, scored 1, 2, 3, or 4 respectively. The TCQ-CA was only administered at T1.

Child Revised Impact of Event Scale (CRIES). The CRIES is a 13 item child and adolescent-appropriate version of the Revised Impact of Event Scale (RIES; Horowitz et al. 1979), a selfreport measure of PTSS in adults. The CRIES contains subscales pertaining to the re-experiencing, avoidance, and hyperarousal symptoms of PTSD. The CRIES has good internal reliability and criterion validity (Perrin et al. 2005).

Trauma-related rumination. A brief three item measure was developed to measure how much adolescents ruminated about the trauma, that is, a perseverative thinking style focused on trying to understand why the trauma had occurred. These items, adapted 
from existing adult measures (Ehlers, et al. 1998), were: "I keep wishing that I could go back in time and prevent the event from happening," "Whenever I think of the event I wonder why it happened to us," and "I am always wondering if my family or I might get hurt again." Participants could respond "never," "sometimes," "often," or "almost always" to the rumination items, scored 1, 2, 3, or 4 respectively. The measure had acceptable internal consistency (Cronbach's $\alpha=0.85$ ). When the study was being designed, it was assumed that rumination would not occur in the acute phase and would take some months to be established; as such, these rumination items were only administered at $\mathrm{T} 2$.

\section{Results}

Eighteen (19.8\%) participants met criteria for ASD at T1, whereas $8(12.3 \%)$ met criteria for PTSD at T2. Distraction was the most frequently endorsed strategy on the TCQ-CA (mean $[\mathrm{M}]=15.51, \mathrm{SD}=3.51)$, then reappraisal $(\mathrm{M}=11.72, \mathrm{SD}=3.40)$, worry $(M=10.54, S D=3.43)$, social support $(M=9.47, S D=3.37$; score doubled here to allow appropriate comparison with the other six item scales), and punishment $(\mathrm{M}=7.89, \mathrm{SD}=2.35)$. Withinsubjects $t$ tests revealed that subscales were endorsed at a significantly different rate to each other.

Scores on each TCQ-CA subscale at T1 and rumination at T2, differentiated by ASD or PTSD status, are displayed in Table 1. No TCQ-CA subscales discriminated between ASD and non-ASD cases, whereas only scores on the punishment subscale of the TCQCA and the rumination measure discriminated PTSD from nonPTSD cases at T2.

The results of correlation analyses are displayed in Table 2. At T1, PTSS (as indexed by the CRIES) were positively associated with the use of punishment, reappraisal, and worry, but not social support or distraction. No subscales were associated with ASD status. The use of punishment, reappraisal, and social support at T1 were also predictive of PTSS at T2, whereas the use of punishment predicted PTSD status at T2. Trauma-related rumination at T2 was significantly correlated with concurrent PTSS and PTSD diagnosis.

Stepwise linear regression was used to investigate the amount of unique variance in PTSS accounted for by thought control variables. Only significant correlates were entered in each model. With T1 PTSS as the dependent variable, reappraisal $(\beta=0.37)$ and punishment $(\beta=0.25)$ were retained in the model, accounting for $28 \%$ of variance $\left(\mathrm{F}_{2,85}=16.35, p<0.0001\right)$. The same variables were retained in a model of PTSS at T2 (reappraisal, $\beta=0.42$; punishment, $\beta=0.29 ; \mathrm{F}_{2,56}=13.03, p<0.0001$; Total $R^{2}=0.32$ ); however, when T1 PTSS was entered in a first step, these variables no longer accounted for any unique variance. Only T2 traumarelated rumination accounted for any variance in T2 PTSS over and above initial PTSS (T1 CRIES, $\beta=0.48$; rumination, $\beta=0.44$; $\mathrm{F}_{2,53}=71.41, p<0.0001$; Total $\left.R^{2}=0.73\right)$.

Mediation analysis was used to explore whether the relationship between punishment and reappraisal thought control strategies at T1 and PTSS at T2 was mediated by rumination (i.e., was increased rumination the pathway by which these thought control strategies in the acute phase led to chronic PTSD). Utilizing the bootstrapping procedure proposed by Hayes and Preacher (2006) to test for mediation effects, rumination was found to fully mediate the relationship between the use of reappraisal at T1 and PTSS at T2 (bootstrap index $=1.77, \mathrm{SE}=0.44,95 \%$ bias-corrected CI $[0.95$, 2.67]), as well as the relationship between the use of punishment at T1 and PTSS at T2 (bootstrap index $=2.90, \mathrm{SE}=0.68,95 \%$ biascorrected CI $[1.46,4.25])$.

\section{Discussion}

The TCQ was amended for use with children and adolescents exposed to trauma. A variety of thought control strategies were used by youth exposed to a recent trauma, with distraction being commonly used, regardless of whether the young person had any significant PTSS. Whereas most measures were not differentiated by ASD or PTSD status, when using a more sensitive continuous measure of PTSS, a number of significant relationships were apparent. Consistent with adult studies (Warda and Bryant 1998; Scarpa et al. 2009; Pietrzak et al. 2011), PTSS was associated with the use of worry- and punishment-based strategies in the acute phase, and the use of punishment even predicted PTSS at a 6 month follow-up assessment. In contrast to adult findings, and at odds with our predictions, the use of reappraisal was associated with greater PTSS in the acute phase, whereas reappraisal and social support were also predictive of later PTSS.

The strongest correlate of PTSS at any point was rumination, which accounted for PTSS at T2, even when accounting for the variance explained by initial PTSS. This relationship is consistent with earlier research in youth (Ehlers et al. 2003; Stallard 2003). However, because of doubt over whether rumination would be established in the acute phase, and concerns about overloading

Table 1. Mean Scores Differentiated by ASD Status at T1 and PTSD Status at T2

\begin{tabular}{|c|c|c|c|c|c|c|c|c|}
\hline & \multicolumn{4}{|c|}{ Time 1} & \multicolumn{4}{|c|}{ Time 2} \\
\hline & $\begin{array}{c}A S D \\
(\mathrm{n}=17) \\
M(S D)\end{array}$ & $\begin{array}{c}\text { Non-ASD } \\
(\mathrm{n}=68) \\
M(S D)\end{array}$ & $\begin{array}{l}\text { Mann- } \\
\text { Whitney } \\
\text { statistic }\end{array}$ & $\mathrm{Z}$ & $\begin{array}{c}P T S D \\
(\mathrm{n}=8) \\
M(S D)\end{array}$ & $\begin{array}{c}\text { Non-PTSD } \\
\quad(\mathrm{n}=49) \\
M(S D)\end{array}$ & $\begin{array}{c}\text { Mann- } \\
\text { Whitney U }\end{array}$ & $\mathrm{Z}$ \\
\hline CRIES total (T1) & $48.56(12.04)$ & $26.17(15.15)$ & 151.5 & $4.69 * *$ & - & - & - & - \\
\hline CRIES total (T2) & - & - & - & - & $43.42(13.26)$ & $18.69(16.02)$ & 50.0 & $3.20 * * *$ \\
\hline TCQ-CA: Distraction & $14.88(4.37)$ & $15.84(3.29)$ & 493.5 & 0.85 & $15.13(4.45)$ & $15.79(3.52)$ & 174.5 & 0.50 \\
\hline TCQ-CA: Punishment & $8.35(2.62)$ & $7.73(2.34)$ & 458.0 & 1.29 & $10.00(2.97)$ & $7.00(1.67)$ & 108.0 & $2.09 *$ \\
\hline TCQ-CA: Social support & $5.06(1.91)$ & $4.72(1.68)$ & 473.5 & 1.6 & $5.25(2.49)$ & $4.49(1.47)$ & 173.5 & 0.53 \\
\hline TCQ-CA: Worry & $11.41(3.95)$ & $10.28(3.25)$ & 473.5 & 1.08 & $11.63(5.40)$ & $10.10(3.09)$ & 171.0 & 0.58 \\
\hline TCQ-CA: Reappraisal & $13.00(3.82)$ & $11.22(3.17)$ & 423.5 & 1.56 & $12.25(3.85)$ & $11.23(3.20)$ & 170.0 & 0.60 \\
\hline Rumination (T2) & - & - & - & - & $9.57(1.72)$ & $6.31(2.52)$ & 59.5 & $2.98 * *$ \\
\hline
\end{tabular}

T1, time 1, 2-4 weeks posttrauma; T2, time 2, 6 months posttrauma.

$* p<0.05, * * p<0.01, * * * p<0.001$.

ASD, acute stress disorder; CRIES, Child Revised Impact of Event Scale; PTSD, posttraumatic stress disorder; TCQ-CA, Thought Control Questionnaire, child and adolescent version. 
Table 2. Correlation Coefficients for Thought Control Strategies and Posttraumatic Stress at Each Assessment Point

\begin{tabular}{|c|c|c|c|c|c|}
\hline \multirow[b]{2}{*}{ Thought control strategy } & \multicolumn{2}{|c|}{$T 1(\mathrm{n}=90)$} & \multicolumn{3}{|c|}{$T 2(\mathrm{n}=61)$} \\
\hline & CRIES & ASD diagnosis & CRIES & PTSD diagnosis & Rumination \\
\hline TCQ-CA - Distraction & -0.04 & -0.09 & -0.02 & -0.07 & 0.00 \\
\hline TCQ-CA - Punishment & $0.42 * *$ & 0.14 & $0.40 * *$ & $0.28 *$ & $0.37 * *$ \\
\hline TCQ-CA - Reappraisal & $0.48 * *$ & 0.17 & $0.49 * *$ & 0.08 & $0.41 * *$ \\
\hline TCQ-CA - Social support & 0.17 & 0.07 & $0.30^{*}$ & 0.07 & 0.25 \\
\hline TCQ-CA - Worry & $0.22 *$ & 0.12 & 0.21 & 0.08 & 0.20 \\
\hline Rumination (T2) & - & - & $0.79 * *$ & $0.38 * *$ & - \\
\hline
\end{tabular}

Spearman's rho coefficients were used because the data were not normally distributed.

T1, time 1, 2-4 weeks posttrauma; T2, time 2, 6 months posttrauma.

$* p<0.05, * * p<0.01$.

ASD, acute stress disorder; CRIES, Child Revised Impact of Event Scale; TCQ-CA, Thought Control Questionnaire, child and adolescent version; PTSD, posttraumatic stress disorder.

participants, this variable was only entered in the T2 assessment battery. As such, it was impossible to establish whether this variable would be temporally predictive as well. Mediation analysis suggested that the impact of both punishment thought control strategies and reappraisal in the acute phase on PTSS at follow-up was brought about through increased levels of rumination.

These findings have several implications. First, these data suggest that children and adolescents' attempts at distraction in the acute phase, although unlikely to be protective against chronic PTSD, are also unlikely to worsen symptoms. As such, distraction may be an acceptable strategy for managing traumarelated distress in the short term. Efforts to suppress thought, however, may result in a paradoxical rebound effects that worsen PTSS (Aaron et al. 1999; Ehlers et al. 2003). Second, children and adolescents must be dissuaded from reacting in a critical or self-punishing way to intrusive phenomena in the acute phase following a trauma. As suggested by cognitive models of PTSD (e.g., Ehlers and Clark 2000), a negative interpretation of intrusions may only serve to heighten distress. Strikingly, this was the only TCQ-CA subscale to demonstrate a difference between PTSD and non-PTSD participants. Furthermore, attempts to think about minor concerns or worries are unlikely to be helpful, and should be cautioned against. Third, the present data sound a note of alarm over the use of social support and reappraisal to manage PTSS. It is possible that the positive correlations observed here simply indicate that more symptomatic youth make greater efforts in the first few weeks following a trauma to seek help, either from others (Prinstein et al. 1996) or through their own powers of reflection. A further possibility is that attempting to use such cognitively demanding strategies for managing PTSS is counterproductive. It may be that younger people lack the skills and maturity to reappraise the situation effectively, or utilize the support of their peers. Indeed, the data presented here suggest that efforts to reappraise trauma-related intrusions - on the face of it an adaptive strategy - may become ruminative and actually worsen PTSS. Fourth, the present study underscores the importance of further understanding rumination in children and adolescents exposed to trauma, how this process emerges following trauma exposure, and how this process may be tackled using psychological interventions.

\section{Conclusions}

The present study is strengthened by the use of a prospective longitudinal design and multi-item measures to assess thought control strategies. It is limited, however, by a modest sample size, a high dropout rate at the follow-up assessment, and the reliance on a questionnaire measure of thought control strategies. Future research is needed to explore the role of thought control strategies in youth, particularly in the acute phase. As well as larger-scale prospective longitudinal studies, experimental research may be of great utility. To date no protective thought control strategies have been identified. It may be that alternative strategies based more on acceptance of intrusions (e.g., as associated with the "third wave" of CBT approaches) and the resumption of typical pre-trauma behavior, rather than efforts at control, may be more effective; future research needs to address these questions.

\section{Clinical Significance}

Health professionals working with young people exposed to traumatic stressors are frequently asked for advice on how to manage the symptoms of PTSD, in particular the re-experiencing symptoms of this disorder. This study looks at the strategies young people use in the first few weeks posttrauma to manage these symptoms (with a focus on intrusive memories), and examines whether any strategies are protective against the development of chronic PTSD. No evidence was found that any strategies are protective against the development of PTSS. It was found that even apparently "positive" ways of handling intrusive memories (e.g., trying to reappraise the traumatic stressor) were associated with worse PTSD symptoms. Such a strategy appears to encourage a ruminative response to the trauma, that is, a preoccupation with the meaning and causes of the trauma, which only heightens anxiety and stress.

\section{Disclosures}

No competing financial interests exist.

\section{References}

Aaron J, Zaglul H, Emery RE: Posttraumatic stress in children following acute physical injury. J Pediatr Psychol 24:335-343, 1999.

Brewin CR, Dalgleish T, Joseph S: A dual representation theory of posttraumatic stress disorder. Psychol Rev 103:670-686, 1996.

Ehlers A, Clark DM: A cognitive model of posttraumatic stress disorder. Behav Res Ther 38:319-345, 2000.

Ehlers A, Mayou RA, Bryant B: Cognitive predictors of posttraumatic stress disorder in children: results of a prospective longitudinal study. Behav Res Ther 41:1-10, 2003. 
Ehlers A, Mayou RA, Bryant B: Psychological predictors of chronic posttraumatic stress disorder after motor vehicle accidents. J Abnorm Psychol 107:508-519, 1998.

Hayes AF, Preacher KJ: Bootstrapping specific indirect effects in multiple mediator models of media effects. Paper presented at the Annual Conference of the Association for Education in Journalism and Mass Communication, San Francisco, CA, 2006.

Horowitz M, Wilner N, Alvarez W: The Impact of Event Scale: A measure of subjective stress. Psychosom Med 41:209-218, 1979.

Meiser-Stedman R, Dalgleish T, Smith P, Yule W, Glucksman E: Diagnostic, demographic, memory quality, and cognitive variables associated with acute stress disorder in children and adolescents. J Abnorm Psychol 116:65-79, 2007.

Perrin S, Meiser-Stedman R, Smith P: The Children's Revised Impact of Event Scale (CRIES): Validity as a screening instrument for PTSD. Behav Cogn Psychother 33:487-498, 2005.

Pietrzak RH, Harpaz-Rotem I, Southwick SM: Cognitive-behavioral coping strategies associated with combat-related PTSD in treatmentseeking OEF-OIF veterans. Psychiatry Res 189:251-258, 2011.

Prinstein MJ, La Greca AM, Vernberg EM, Silverman WK: Children's coping assistance: How parents, teachers, and friends help children cope after a natural disaster. J Clin Child Psychol 25:463-475, 1996.

Reynolds M, Wells A: The Thought Control Questionnairepsychometric properties in a clinical sample, and relationships with PTSD and depression. Psychol Med 29:1089-1099, 1999.
Scarpa A, Wilson LC, Wells AO, Patriquin MA, Tanaka A: Thought control strategies as mediators of trauma symptoms in young women with histories of child sexual abuse. Behav Res Ther 47: 809-813, 2009.

Silverman WK, Albano AM: Anxiety Disorder Interview Schedule for DSM-IV: Child and Parent Interview Schedule. San Antonio, TX: The Psychological Corporation; 1996.

Stallard P: A retrospective analysis to explore the applicability of the Ehlers and Clark (2000) cognitive model to explain PTSD in children. Behav Cogn Psychother 31:337-345, 2003.

Warda G, Bryant RA: Thought control strategies in acute stress disorder. Behav Res Ther 36:1171-1175, 1998

Wells A, Davies MI: The Thought Control Questionnaire: A measure of individual differences in the control of unwanted thoughts. Behav Res Ther 32:871-878, 1994.

Address correspondence to: Richard Meiser-Stedman, PhD MRC Cognition \& Brain Sciences Unit 15 Chaucer Road Cambridge, CB2 7EF United Kingdom

E-mail: richard.meiser-stedman@mrc-cbu.cam.ac.uk 